\title{
Total antioxidant capacity-Relevance, methods and clinical implications
}

\author{
Sajal Gupta ${ }^{1}$ (D) | Renata Finelli ${ }^{1}$ (D) $\mid$ Ashok Agarwal $^{1}$ (D) | Ralf Henkel ${ }^{1,2}$ (D)
}

\author{
${ }^{1}$ American Center for Reproductive \\ Medicine, Cleveland Clinic, Cleveland, $\mathrm{OH}$, \\ USA \\ ${ }^{2}$ Department of Medical Bioscience, \\ University of the Western Cape, Bellville, \\ South Africa \\ Correspondence \\ Ashok Agarwal, American Center for \\ Reproductive Medicine, Cleveland Clinic, \\ Mail Code X-11, 10681 Carnegie Avenue, \\ Cleveland, OH 44195, USA. \\ Email: agarwaa@ccf.org
}

\begin{abstract}
Oxidative stress is pre-empted by an adequate level of antioxidants, which scavenge oxidants when they are produced in excess by different sources, including leukocytes and immature spermatozoa. Enzymatic antioxidants, such as superoxide dismutase, catalase and glutathione peroxidase, and several non-enzymatic antioxidants (proteins, vitamins and minerals), working as oxidant scavengers and cofactors of enzymatic antioxidants have been identified in seminal plasma. The total antioxidant capacity (TAC) is a diagnostic test that can be utilised in the male infertility workup. TAC measures the amount of total antioxidants in seminal plasma. Therefore, it provides an assessment of the reductive potential in seminal plasma. Several studies have investigated the diagnostic application of TAC in various andrology conditions. There is substantial evidence in the literature to show that infertile patients have lower seminal TAC in comparison with fertile men. Moreover, there is a positive correlation between TAC and seminal parameters, such as sperm concentration, motility and morphology. Evaluation of TAC together with reactive oxygen species (ROS) and sperm DNA fragmentation index (DFI) may be beneficial in the diagnosis of male infertility.
\end{abstract}

\section{KEYWORDS}

antioxidants, male infertility, oxidative stress, reactive oxygen species, spermatozoa

\section{1 | BACKGROUND}

In seminal fluid, oxidants and antioxidants are secreted by different cell types. In particular, leukocytes and immature spermatozoa represent the main source of reactive oxygen species (ROS). In order to accomplish the various physiological processes, the redox balance is maintained by an equitable ratio between oxidants and antioxidants (Figure 1). On the one hand, a physiological ROS concentration is involved in the regulation of many physiological sperm processes such as capacitation, acrosome reaction, acquisition of hyperactivation and sperm-oocyte membrane fusion (Aitken, 2017). However, when ROS are generated in excess or in case of limited antioxidant concentrations, there can be a shift in the redox potential towards oxidative stress.
This article focuses on antioxidants and their role in balancing the physiological seminal redox potential. It discusses the evaluation of total antioxidant capacity (TAC), as a parameter to evaluate oxidative stress, and the advantages and disadvantages of each methodology for antioxidant assessment. The functional impact of the redox imbalance and lower antioxidants levels on sperm parameters and their clinical implications in various male infertility conditions are described.

\section{1 | Antioxidant defence in semen}

Antioxidant defences in the ejaculate are localised in spermatozoa and seminal plasma. However, during maturation, spermatozoa 
undergo several morphological changes, resulting in the loss of cytoplasm and nuclear DNA compaction. Therefore, their cytoplasmic antioxidant fraction is limited, and spermatozoa rely on antioxidants in seminal plasma to counteract oxidative stress. These can be generally classified as enzymatic and non-enzymatic antioxidants.

\subsection{1 | Enzymatic antioxidants}

Superoxide dismutase (SOD), catalase (CAT) and glutathione peroxidase (GPx) are the main enzymatic antioxidants in semen. They work synergistically, as SOD converts the superoxide anion $\left(\mathrm{O}_{2}^{-}\right)$ into $\mathrm{H}_{2} \mathrm{O}_{2}$. CAT and GPx, in turn, convert $\mathrm{O}_{2}^{-}$into molecular oxygen or alcohols, respectively, contributing to the removal of oxidants (Kehrer, 2000; Valko, Izakovic, Mazur, Rhodes, \& Telser, 2004). In the male reproductive tract, three different SOD isoforms are produced, thereby contributing to antioxidants scavenging: (a) the homodimeric SOD1 is a Cu/Zn-binding enzyme which localises at the level of mitochondria and cytosol; (b) the mitochondrial homotetrameric SOD2 binds to manganese and it is inhibited by high levels of peroxynitrite (MacMillan-Crow \& Thompson, 1999); and (c) the extracellular homotetrameric Cu/Zn SOD3 isoform has been described in extracellular fluids of eukaryotes. They are expressed in the testis by Sertoli, Leydig and germ cells and in the epididymal head and body (Bauché, Fouchard, \& Jégou, 1994; Mruk et al., 1998). Lower levels of CAT have been detected along the epididymal tract and in the testis of rats (Mendis-Handagama, Zirkin, Scallen, \& Ewing, 1990; Peltola, Huhtaniemi, \& Ahotupa, 1992; Zini $\&$ Schlegel, 1997). Spermatozoa show CAT activity but such activity has not been reported in the other stages of sperm development (Bauché et al., 1994). Several selenium-dependent and seleniumindependent isoforms of GPx have been described as cytoplasmic, membrane-bound or secreted proteins. They work in concert with glutathione: the latter acts as electron donor to catalyze the reduction of $\mathrm{H}_{2} \mathrm{O}_{2}$ in $\mathrm{H}_{2} \mathrm{O}$. Several isoforms, such as GPx3 and GPx5, are reportedly expressed in the murine epididymal head (Schwaab et al., 1995; Zini \& Schlegel, 1997), where they bind on the surface of spermatozoa, Sertoli and Leydig cells (Luo et al., 2006). In germ cells, GPx enzymatic activity has been reported at various stages of spermatogenesis, from spermatogonia to mature spermatozoa, albeit at low levels (Bauché et al., 1994).

\subsection{2 | Non-enzymatic antioxidants}

Several non-enzymatic antioxidants have been identified in seminal plasma, such as proteins, vitamins and minerals, working as oxidant scavengers and cofactors of enzymatic antioxidants. Selenium and glutathione are some of the most abundant antioxidants, and they work as cofactors in concert with GPx isoforms (Majzoub \& Agarwal, 2018). Low levels of glutathione are synthesised in Sertoli and Leydig cells, with a decline in levels with increasing age (Luo et al., 2006). Glutathione is highly expressed in spermatocytes and spermatids but less in matured spermatozoa (Bauché et al., 1994). Zinc is another mineral showing antioxidant properties based on its capability to neutralise the actions of transition metals such as iron and copper (Powell, 2000). Vitamins, such as the fat-soluble tocopherol (vitamin E), protect spermatozoa from oxidative stress, counteracting the lipid peroxidation of sperm membranes (Suleiman, Elamin Ali, Zaki, El-Malik, \& Nasr, 1996). In the same way, water-soluble compounds, such as ascorbic acid (vitamin C) and L-carnitine, protect spermatozoa from the lipid and DNA damage induced by oxidative stress (Majzoub \& Agarwal, 2018). Coenzyme Q10 is a quinone expressed in sperm mitochondria and is directly involved in the generation of energy (Majzoub \& Agarwal, 2018). Therefore, it acts as a scavenger of oxidants produced during oxidative phosphorylation along the electron transport chain system. Lycopene is an organic compound identified in the testis, specifically in Sertoli cells and round spermatids (Ferreira et al., 2000; Rao, Ray, \& Rao, 2006). It is consumed in diet, including vegetables and fruits which are rich in this pigment (Durairajanayagam, Agarwal, Ong, \& Prashast, 2014). Other non-enzymatic antioxidants present at lower concentration include uric acid, vitamin B9, $\beta$-carotene, hypotaurine and taurine.

\section{Oxidative stress}
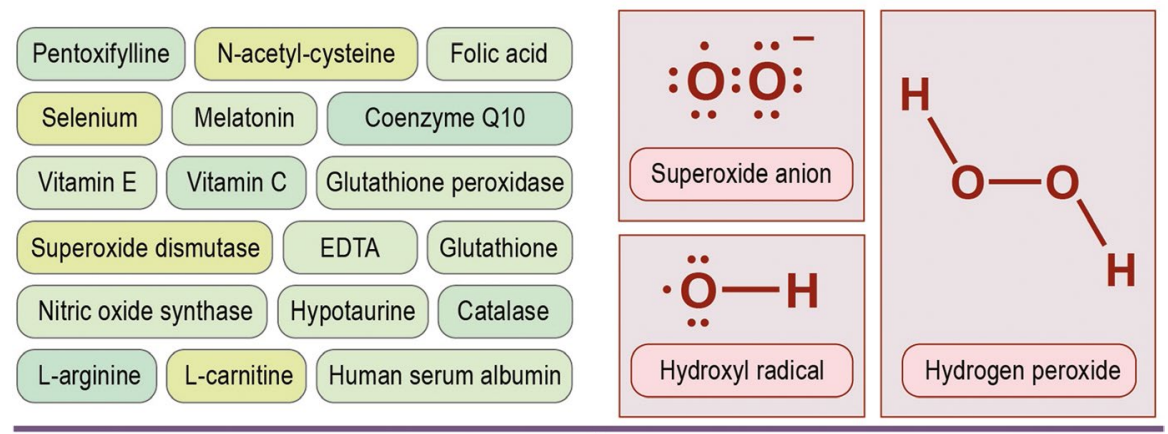

Antioxidants

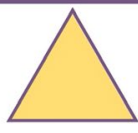

ROS
FIGURE 1 Oxidative stress results from a disequilibrium between antioxidants and reactive oxygen species (ROS) 


\section{2 | MECHANISMS BY WHICH ANTIOXIDANTS HELP PROTECT THE SPERMATOZOA}

Along their journey from the testis to the female reproductive tract, spermatozoa are subjected to numerous insults which can limit their fertilising potential. Oxidative stress and ROS generation have been broadly observed in male infertile patients, as they damage spermatozoa structurally and functionally. ROS are able to react with any molecular component, triggering the generation of new radical molecules in a self-perpetuating oxidative stress cycle resulting in cellular damage.

Extracellular oxidants are responsible for lipid peroxidation of sperm membrane, impacting sperm motility and morphology. Intracellular ROS can react with proteins, altering the physiological pathways by oxidation of substrates, and DNA, causing sperm DNA fragmentation (Figure 2). Therefore, antioxidants play an essential role in balancing the seminal concentration of oxidants in sperm physiology. The protection from oxidative stress is achieved in three steps, as described in the following sections.

\section{1 | Prevention}

Spermatozoa use several strategies to prevent the ROS-mediated cellular damage. During the process of spermiogenesis, genomic DNA is strictly compacted around protamines, shielding the DNA from the action of oxidants. Moreover, the mitochondria, which are the main sources of cellular ROS, are localised in the mid-piece and are spatially far from the nucleus. This limits the access to sperm DNA by ROS such as $\mathrm{O}_{2}^{-}$, which cannot cross the membranes with lipids as the predominant molecular component. Several enzymes contribute to ROS generation with their catalytic action. However, enzymatic proteins can be tridimensional and folded in a way that prevents the release of generated ROS during their enzymatic activity. The enzyme cytochrome oxidase, for example, is the last component of the electron transport chain. It is associated with iron and copper ions and catalyses the reduction of molecular oxygen into $\mathrm{H}_{2} \mathrm{O}$. Nevertheless, the composition of this hetero-oligomeric enzyme prevents the ROS release in the intracellular milieu (Bourens, Fontanesi, Soto, Liu, \& Barrientos, 2013).

\section{2 | Interception}

Enzymatic and non-enzymatic systems are involved in the interception, or deactivation, of oxidant species, in order to limit their toxic activity. Furthermore, they delocalise oxidants in different intracellular compartments, such as the membrane and the aqueous seminal plasma, where their effects are less harmful. Phenolic compounds, such as $\alpha$-tocopherol, work as antioxidants, protecting the membranes from lipid peroxidation. Additionally, they react with ascorbic acid and thiols to regenerate their reductive potential (Sies, 1993). The ROS half-life influences the scavenger capability of antioxidant systems: peroxyl radicals, having a half-life of $\sim 7 \mathrm{~s}$, are more likely to be deactivated than hydroxyl radical $\left(10^{-9} \mathrm{~s}\right)$ (Sies, 1993).
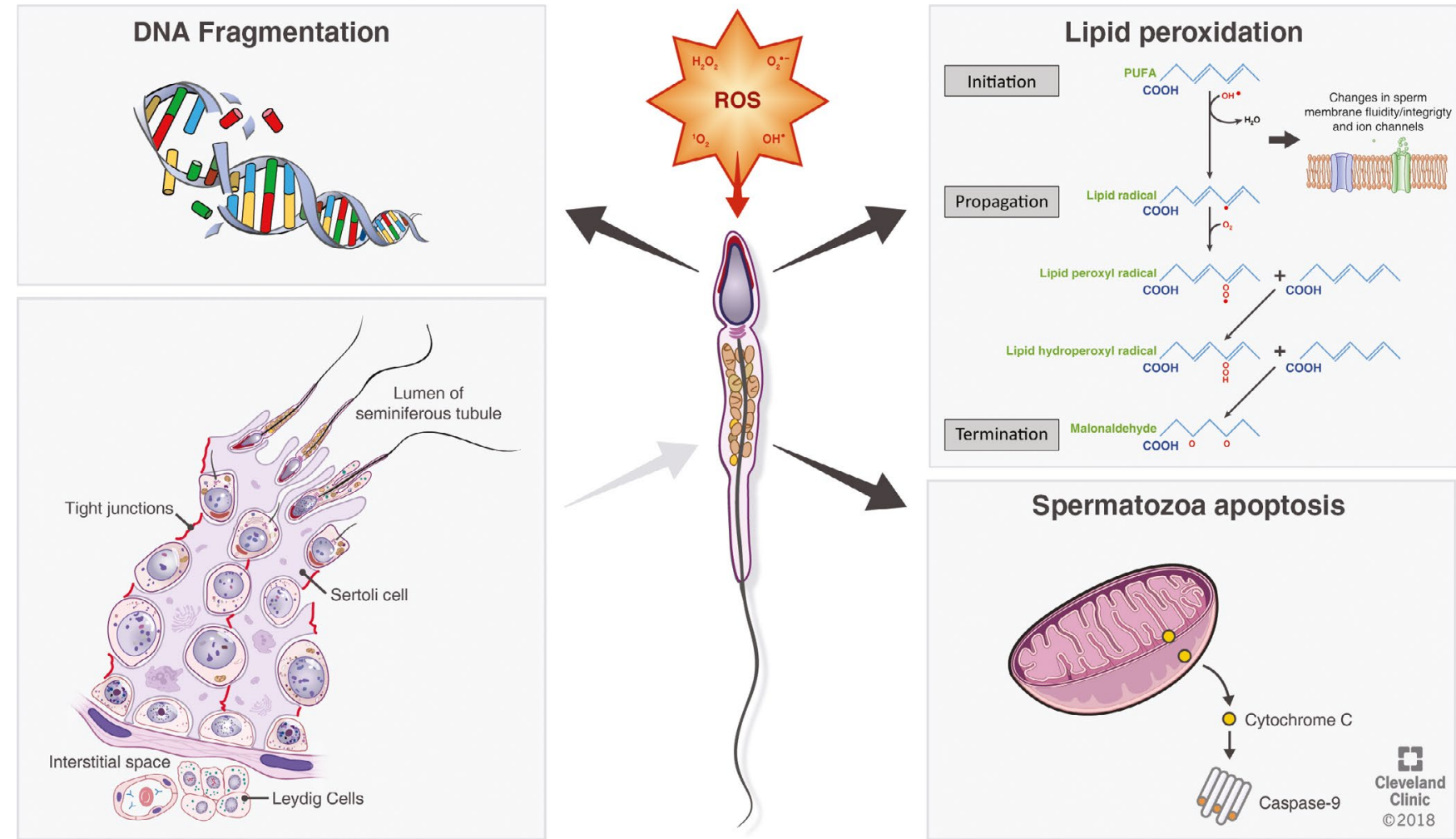

FIGURE 2 Mechanisms of ROS-mediated sperm damage 


\section{3 | Repair}

Following the ROS-induced sperm damage, the outcomes are determined by the severity of the damage. The activation of the apoptotic pathway occurs in a setting of severe damage, resulting in the elimination of damaged spermatozoa. If the damage is milder, it is repaired by the oocyte. The repair mechanisms of the oxidative stress-related damage are very limited in spermatozoa, as they are transcriptionally and translationally silent cells (González-Marín, Gosálvez, \& Roy, 2012). Specifically, no mechanisms to repair DNA damage are available once spermiogenesis is completed. This increases the susceptibility of mature spermatozoa to the oxidative stress-mediated damage. Moreover, mature spermatozoa only have a small amount of cytoplasm and are therefore dependent on the seminal plasma for antioxidant defences.

\section{3 | ANTIOXIDANT CAPACITY AND SPERM FUNCTION}

Low antioxidant levels in seminal plasma lead to oxidative stress, which is an important causative factor in idiopathic male infertility. Elevated levels of ROS affect the sperm structure and functions and these, in turn, lead to reproductive disorders such as fertilisation failure or spontaneous miscarriage and/ or recurrent miscarriages (Kamkar, Ramezanali, \& Sabbaghian, 2018; Opuwari \& Henkel, 2016).

Sperm parameters such as sperm count, motility and morphology have been reported to show a strong positive correlation with the levels of Zinc and TAC, both being significantly lower in infertile men (Badade, More, Narshetty, Badade, \& Yadav, 2011). TAC levels have been reported to be significantly lower in men whose partners had repeated pregnancy loss when compared to fertile men (Kamkar et al., 2018). Bassiri et al. reported a significant correlation between abnormal sperm function tests, such as higher numbers of chromomycin A3 positive spermatozoa and lipid peroxidation levels in infertile men with one failed ICSI cycle. This indicates that this group of men may benefit from antioxidant supplementation (Bassiri, Nasr-Esfahani, Forozanfar, \& Tavalaee, 2020).

\section{4 | MEASUREMENT OF TOTAL ANTIOXIDANT CAPACITY}

Total antioxidant capacity is a diagnostic test that can be used in the male infertility workup. TAC measures the amount of total antioxidants in seminal plasma. Therefore, it evaluates oxidative stress, providing an assessment of the reductive potential in seminal plasma. The same test can be performed using different approaches which are presented in Table 1.

\subsection{Sample collection}

The sample is collected by masturbation in a clean plastic cup after 2-7 days of sexual abstinence, according to the most recent $\mathrm{WHO}$ guidelines (2010) (WHO, 2010). It is important to collect the entire sample without losing any part, and when the sample is collected at home, it must be kept at body temperature during the transport and delivered no later than $1 \mathrm{hr}$. Once the sample is liquefied, it is centrifuged at $300 \mathrm{~g}$ for $10 \mathrm{~min}$, in order to remove the cell fraction and obtain a clear seminal plasma, which can be tested for TAC.

\section{2 | Methods for assessing TAC: principles, methodology and reference values}

Several methods for the assessment of seminal TAC have been proposed in the literature (Table 1). They vary according to the principle and methodology as well as their sensitivity to different antioxidants. Therefore, results may vary according to the type of test that is used (Vassalle et al., 2004).

\subsection{1 | 2,2-Diphenyl-1-Picrylhydrazyl (DPPH)}

DPPH provides a spectrophotometric evaluation of TAC at a wavelength of $515 \mathrm{~nm}$. At room temperature and in alcohol solutions, such

\begin{tabular}{|l|l|}
\hline Technique & Principle \\
\hline 2,2-Diphenyl-1-Picrylhydrazyl (DPPH) & $\begin{array}{l}\text { Spectrophotometric evaluation of reduced } \\
\text { purple-coloured DPPH }\end{array}$ \\
\hline Trolox equivalent antioxidant capacity (TEAC) & $\begin{array}{l}\text { Spectrophotometric evaluation of oxidised } \\
\text { blue/green-coloured 2,29-azinobis-(3-ethyl } \\
\text { benzothiazoline-6-sulphonic acid) (ABTS) }\end{array}$ \\
\hline TEAC assay ELISA assay & $\begin{array}{l}\text { Measurement of oxidised ABTS by ELISA } \\
\text { assay }\end{array}$ \\
\hline Ferric reducing antioxidant power (FRAP) & $\begin{array}{l}\text { Spectrophotometric evaluation of the } \\
\text { reduction of the ferric tripyridyltriazine } \\
\text { (Fe } \\
\text { analogue (Fe }{ }^{2+} \text { - TPTZ) }\end{array}$ \\
\hline Enhanced chemiluminescence (ECL) assay & $\begin{array}{l}\text { Oxidation of luminol and the generation of } \\
\text { a chemiluminescence signal, detected by a } \\
\text { luminometer }\end{array}$ \\
\hline
\end{tabular}

TABLE 1 Techniques used to evaluate the total antioxidant capacity of seminal plasma 
as methanol, ethanol or other organic solvents, DPPH is stable and purple-coloured. On the other hand, in the presence of antioxidants, DPPH is quickly reduced to 1,1-diphenyl-2-picrylhydrazine and thereby loses its colour. Therefore, when the sample is incubated in DPPH solution, the resulting colorimetric change is inversely proportional to the amount of antioxidants in the sample (Garcia et al., 2012). The results, expressed as percentage, are calculated considering the absorbance values of samples and control (absorbance value obtained by the incubation of DPPH in methanol without the sample), according to the following formula: [(Abs CTRL - Abs sample): Abs CTRL] × 100 (Subramanian et al., 2018).

In a recent study published in 2018 involving 87 subfertile men and 23 fertile men, a cut-off of $77.4 \%$ of the TAC levels was proposed to discriminate patients according to their fertility status with moderate sensitivity (69\%) and specificity (58\%) (Subramanian et al., 2018).

\subsection{2 | Trolox equivalent antioxidant capacity (TEAC)}

The concentration of antioxidants is assessed with a spectrophotometer which evaluates the synthesis of a blue/green-coloured oxidised substrate (Rhemrev et al., 2000).

In this technique, described by Miller, Rice-Evans, Davies, Gopinathan, and Milner (1993), the compound 2,29-azinobis-(3-ethyl benzothiazoline-6-sulphonic acid) (ABTS) is oxidised to $\mathrm{ABTS}^{+}$, after being incubated with metmyoglobin and $\mathrm{H}_{2} \mathrm{O}_{2}$. This coloured substrate can be quantified spectrophotometrically at a wavelength of $750 \mathrm{~nm}$. The colorimetric signal is compared with that generated by a standard curve of Trolox (6-hydroxy-2,5,7, 8-tetramethylchroman-2-carboxylic acid), an analogue of tocopherol. The antioxidants concentration is obtained indirectly based on its capability to inhibit the oxidation of ABTS and to reduce the colour of the solution. Results are provided as micromoles of Trolox equivalent.

In a study conducted in 2016, Roychoudhury, Sharma, Sikka, and Agarwal (2016) analysed TAC in a group of 279 infertile patients and 46 fertile men with the aim to identify a putative cut-off value to discriminate the patients according to their oxidative seminal status. A cut-off value $\geq 1,950 \mu \mathrm{M}$ Trolox was proposed, showing relatively low specificity (63.0\%) and sensitivity (59.5\%) (Roychoudhury et al., 2016).

\subsection{3 | Assay-based on formation of ABTS radical: ELISA assay}

The formation of ABTS radical in TEAC assay is usually detected spectrophotometrically. However, an enzyme-linked immunosorbent assay (ELISA) can be performed. Although the principle of the technique remains the same, the technology used for the detection of ABTS+ radical differs. An ELISA reader is equipped with a variable number of filters to monitor the standard wavelengths. It allows the analysis of multiple samples in a microplate, whereas a spectrophotometer analyses one sample at a time and requires more time and a larger volume of sample.

\subsubsection{Oxygen radical absorbance capacity (ORAC)}

ORAC assay, elaborated by Glazer (1990), is based on the capability of antioxidants to inhibit the oxidation of substrates by exogenous oxidants.

The 2,2'-azobis-(2-amidino-propane) dihydrochloride (AAPH) is a powerful oxidant molecule that can induce the synthesis of peroxyl radicals (Cao \& Prior, 1999). Fluorescent probes, such as B-phycoerythrin, fluorescein and $\mathrm{H}_{2}$ DCFDA, are used to detect peroxyl radicals. Specifically, when they bind to the probes, the fluorescence is reduced. Therefore, the antioxidant concentration is inversely correlated with the decrease in the fluorescence observed.

\subsection{5 | Spectrophotometric assay: ferric reducing antioxidant power (FRAP)}

The FRAP assay, as described by Benzie and Strain (1996), is a direct test to assess the TAC based on the reduction of the ferric tripyridyltriazine $\left(\mathrm{Fe}^{3+}-\mathrm{TPTZ}\right)$ to the ferrous analogue $\left(\mathrm{Fe}^{2+}\right.$ - TPTZ) by antioxidants at low pH (Prior \& Cao, 1999). In fact, antioxidants are able to reduce such molecules the same way as they reduce ROS. Therefore, the generation of $\mathrm{Fe}^{2+}-$ TPTZ, a bluecoloured molecule, is used as an indicator of antioxidant potential in seminal plasma and it can be easily analysed spectrophotometrically at a wavelength of $593 \mathrm{~nm}$. However, FRAP evaluation does not include molecules which cannot reduce $\mathrm{Fe}^{3+}$ - TPTZ, like glutathione, an antioxidant in vivo (Prior \& Cao, 1999). Results are expressed as $\mu \mathrm{mol} / \mathrm{L}$, calculated in comparison with a calibration curve created with a standard dilution of $\mathrm{FeSO}_{4} 7 \mathrm{H}_{2} \mathrm{O}$ (Vassalle et al., 2004).

\subsubsection{Enhanced chemiluminescence $(E C L)$ assays}

This test evaluates the concentration of non-enzymatic antioxidants in seminal plasma based on the capacity of endogenous antioxidants to limit the generation of a chemiluminescent signal (Said et al., 2003).

As described by Alho and Leinonen in detail (Alho \& Leinonen, 1999), the reactive solution, horseradish peroxidase (HRP) linked immunoglobulins and exogenous $\mathrm{H}_{2} \mathrm{O}_{2}$ catalyse the oxidation of luminol and the generation of a chemiluminescence signal. The addition of para-iodophenol to the solution ensures a stable chemiluminescent light. Antioxidants present in the seminal plasma counteract the generation of chemiluminescence, and the reduction of the signal indirectly provides assessment of the antioxidant concentration in the 
fluid. In fact, like in TEAC assay, a quantitative measurement is done by comparison of the signal obtained with that generated by a standard curve of Trolox. Results are provided as molar Trolox equivalent. The evaluation of TAC by chemiluminescence takes around 45 min and requires a sophisticated and expensive luminometer.

\section{5 | CLINICAL DIAGNOSTIC VALUE OF ANTIOXIDANT CAPACITY}

Several studies have investigated the diagnostic application of TAC in andrology. Literature reports that infertile patients have lower seminal TAC in comparison with fertile men. Furthermore, a positive correlation between TAC and seminal parameters, such as sperm concentration, motility and morphology, has been reported (Eroglu et al., 2014; Mahfouz, Sharma, Sharma, Sabanegh, \& Agarwal, 2009; Roychoudhury et al., 2016; Subramanian et al., 2018). TAC levels were significantly lower in idiopathic male infertility (Eroglu et al., 2014), in astheno- and oligoasthenoteratozoospermic patients (Colagar, Karimi, \& Jorsaraei, 2013; Pahune, Choudhari, \& Muley, 2013), in male partners of couples experiencing idiopathic repeated pregnancy loss (Kamkar et al., 2018) and varicocele patients (Giulini, 2009). Evaluation of TAC in male infertility can provide useful information in the decision of treatment. For instance, surgical treatment of varicocele was demonstrated to improve the seminal redox environment with a significant increase of TAC levels, as observed in case of moderate and highgrade varicocele (Ozturk et al., 2012). In addition, the evaluation of antioxidant concentration can provide information about the seminal redox status and identify those patients who could benefit from antioxidant supplementation. In fact, some studies reported an increase of TAC in asthenoteratozoospermic and oligoasthenoteratozoospermic patients following supplementation of alphalipoic acid and zinc sulphate/folic acid, respectively (Haghighian, Haidari, Mohammadi-Asl, \& Dadfar, 2015; Raigani et al., 2014). Moreover, the evaluation of TAC can be informative of the seminal oxidative status in patients exposed to environmental factors which can affect spermatogenesis. Patients exposed to high levels of ionising radiations as well as lead showed increased seminal TAC value in comparison with controls, as a compensative mechanism in response to the damage (Kasperczyk, Dobrakowski, Czuba, Horak, \& Kasperczyk, 2015; Kumar et al., 2014). However, increased pregnancy rate has not yet been seen based on improvement in TAC.

\section{1 | ROS-TAC score and its predictive value in discriminating fertile from infertile men}

Recently, there has been an increasing number of reports indicating oxidative stress as a causative factor of idiopathic male infertility (Agarwal et al., 2019). Sharma, Pasqualotto, Nelson, Thomas, and Agarwal (1999) suggested a new composite ROS-TAC score for the evaluation of seminal oxidative stress based on the principal component analysis (Sharma et al., 1999). They analysed the predictive power of the ROS-TAC score in comparison with TAC or ROS alone. A ROS-TAC value of $50.00 \pm 10.00$ was reported in fertile men, significantly higher than ROS-TAC observed in patients with varicocele, varicocele and prostatitis, and idiopathic infertility. Moreover, the ROS-TAC score showed a higher power to discriminate patients according to their fertility status than did the ROS or TAC parameters alone (Sharma et al., 1999). ROS-TAC values $<50.00 \pm 10.00$ have been reported in patients with varicocele (Saleh, Agarwal, Sharma, Nelson, \& Thomas, 2002; Saleh et al., 2003) or chronic prostatitis (Pasqualotto et al., 2000), as well as in asthenozoospermic (Vatannejad et al., 2017) and idiopathic infertile patients (Pasqualotto et al., 2001; Pasqualotto, Sharma, Pasqualotto, \& Agarwal, 2008) or in association with lifestyle factors such as smoking (Saleh et al., 2002),

\section{6 | CONCLUSION}

Oxidative stress is a well-established cause of male infertility and its evaluation is of great importance in the workup of male fertility. Since oxidative stress arises as a result of the disequilibrium between pro- and antioxidant molecules, the evaluation of TAC provides information on the redox status of seminal samples. Moreover, it highlights the reductive power of endogenous seminal antioxidants to counterbalance ROS generation in several clinical andrological conditions associated with oxidative stress.

\section{7 | TAKE-HOME MESSAGE}

- Antioxidants play an important role in counterbalancing the ROS and their impact on sperm functions and parameters.

- The evaluation of TAC is informative of the seminal redox status and can help individualise patient management and selection of patients for optimal results with antioxidant supplementation.

- In the workup of male infertility, the evaluation of ROS-TAC score has a higher prognostic capacity than do the ROS or TAC parameters individually.

\section{ORCID}

Sajal Gupta iD https://orcid.org/0000-0001-6487-9681

Renata Finelli iD https://orcid.org/0000-0002-5926-6407

Ashok Agarwal iD https://orcid.org/0000-0003-0585-1026

Ralf Henkel iD https://orcid.org/0000-0003-1128-2982

\section{REFERENCES}

Agarwal, A., Parekh, N., Panner Selvam, M. K., Henkel, R., Shah, R., Homa, S. T., ... Harlev, A. (2019). Male oxidative stress infertility (MOSI): Proposed terminology and clinical practice guidelines for management of idiopathic male infertility. The World Journal of Men's Health, 37(3), 296-312. https://doi.org/10.5534/wjmh.190055 
Aitken, R. (2017). Reactive oxygen species as mediators of sperm capacitation and pathological damage. Molecular Reproduction and Development, 84(10), 1039-1052. https://doi.org/10.1002/ $\operatorname{mrd} .22871$

Alho, H., \& Leinonen, J. (1999). Total antioxidant activity measured by chemiluminescence methods. Methods in Enzymology, 299, 3-15.

Badade, Z., More, K., Narshetty, J., Badade, V., \& Yadav, B. (2011). Human Seminal oxidative stress: Correlation with antioxidants and sperm quality parameters. Annals of Biological Research, 2(5), 351-359.

Bassiri, F., Nasr-Esfahani, M. H., Forozanfar, M., \& Tavalaee, M. (2020). Relationship between sperm parameters with sperm function tests in infertile men with at least one failed cycle after intracytoplasmic sperm injection cycle. International Journal of Fertility and Sterility, 13(4), 324-329.

Bauché, F., Fouchard, M. H., \& Jégou, B. (1994). Antioxidant system in rat testicular cells. FEBS Letters, 349(3), 392-396. https://doi. org/10.1016/0014-5793(94)00709-8

Benzie, I. F. F., \& Strain, J. J. (1996). The ferric reducing ability of plasma (FRAP) as a measure of "antioxidant power": The FRAP assay. Analytical Biochemistry, 239(1), 70-76. https://doi.org/10.1006/ abio.1996.0292

Bourens, M., Fontanesi, F., Soto, I. C., Liu, J., \& Barrientos, A. (2013). Redox and reactive oxygen species regulation of mitochondrial cytochrome C oxidase biogenesis. Antioxidants and Redox Signaling, 19(16), 1940-1952.

Cao, G., \& Prior, R. L. (1999). Measurement of oxygen radical absorbance capacity in biological samples. Methods in Enzymology, 299, 50-62.

Colagar, A. H., Karimi, F., \& Jorsaraei, S. G. A. (2013). Correlation of sperm parameters with semen lipid peroxidation and total antioxidants levels in astheno- and oligoasheno- teratospermic men. Iranian Red Crescent Medical Journal, 15(9), 780-785.

Durairajanayagam, D., Agarwal, A., Ong, C., \& Prashast, P. (2014). Lycopene and male infertility. Asian Journal of Andrology, 16(3), 420425. https://doi.org/10.4103/1008-682X.126384

Eroglu, M., Sahin, S., Durukan, B., Ozakpinar, O. B., Erdinc, N., Turkgeldi, L., ... Karateke, A. (2014). Blood serum and seminal plasma selenium, total antioxidant capacity and coenzyme Q10 levels in relation to semen parameters in men with idiopathic infertility. Biological Trace Element Research, 159(1-3), 46-51. https://doi.org/10.1007/s1201 1-014-9978-7

Ferreira, A., Yeum, K., Liu, C., Smith, D., Krinsky, N., Wang, X., \& Russell, R. (2000). Tissue distribution of lycopene in ferrets and rats after lycopene supplementation. The Journal of Nutrition, 130(5), 12561260. https://doi.org/10.1093/jn/130.5.1256

Garcia, E., Oldoni, T., de Alencar, S., Reis, A., Loguercio, A., \& Grande, R. (2012). Antioxidant activity by DPPH assay of potential solutions to be applied on bleached teeth. Brazilian Dental Journal, 23(1), 22-27. https://doi.org/10.1590/S0103-64402012000100004

Giulini, S., Sblendorio, V., Xella, S., La Marca, A., Palmieri, B., \& Volpe, A. (2009). Seminal plasma total antioxidant capacity and semen parameters in patients with varicocele. Reproductive BioMedicine Online, 18(5), 617-621. https://doi.org/10.1016/S1472-6483(10)60004-1

Glazer, A. N. (1990). Phycoerythrin fluorescence-based assay for reactive oxygen species. Methods in Enzymology, 186(C), 161-168.

González-Marín, C., Gosálvez, J., \& Roy, R. (2012). Types, causes, detection and repair of DNA fragmentation in animal and human sperm cells. International Journal of Molecular Sciences, 13, 14026-14052. https://doi.org/10.3390/ijms131114026

Haghighian, H. K., Haidari, F., Mohammadi-Asl, J., \& Dadfar, M. (2015). Randomized, triple-blind, placebo-controlled clinical trial examining the effects of alpha-lipoic acid supplement on the spermatogram and seminal oxidative stress in infertile men. Fertility and Sterility, 104(2), 318-324. https://doi.org/10.1016/j.fertnstert.2015.05.014

Kamkar, N., Ramezanali, F., \& Sabbaghian, M. (2018). The relationship between sperm DNA fragmentation, free radicals and antioxidant capacity with idiopathic repeated pregnancy loss. Reproductive Biology, 18(4), 330-335. https://doi.org/10.1016/j. repbio.2018.11.002

Kasperczyk, A., Dobrakowski, M., Czuba, Z. P., Horak, S., \& Kasperczyk, S. (2015). Environmental exposure to lead induces oxidative stress and modulates the function of the antioxidant defense system and the immune system in the semen of males with normal semen profile. Toxicology and Applied Pharmacology, 284(3), 339-344. https:// doi.org/10.1016/j.taap.2015.03.001

Kehrer, J. P. (2000). The Haber-Weiss reaction and mechanisms of toxicity. Toxicology, 149(1), 43-50. https://doi.org/10.1016/S0300 $-483 \times(00) 00231-6$

Kumar, D., Salian, S. R., Kalthur, G., Uppangala, S., Kumari, S., Challapalli, S., ... Adiga, S. K. (2014). Association between sperm DNA integrity and seminal plasma antioxidant levels in health workers occupationally exposed to ionizing radiation. Environmental Research, 132, 297304. https://doi.org/10.1016/j.envres.2014.04.023

Luo, L., Chen, H., Trush, M., Show, M., Anway, M., \& Zirkin, B. (2006). Aging and the brown norway rat leydig cell antioxidant defense system. Journal of Andrology, 27(2), 240-247. https://doi.org/10.2164/ jandrol.05075

MacMillan-Crow, L., \& Thompson, J. (1999). Tyrosine modifications and inactivation of active site manganese superoxide dismutase mutant (Y34F) by peroxynitrite. Archives of Biochemistry and Biophysics, 366(1), 82-88. https://doi.org/10.1006/abbi.1999.1202

Mahfouz, R., Sharma, R., Sharma, D., Sabanegh, E., \& Agarwal, A. (2009). Diagnostic value of the total antioxidant capacity (TAC) in human seminal plasma. Fertility and Sterility, 91(3), 805-811. https://doi. org/10.1016/j.fertnstert.2008.01.022

Majzoub, A., \& Agarwal, A. (2018). Systematic review of antioxidant types and doses in male infertility: Benefits on semen parameters, advanced sperm function, assisted reproduction and live-birth rate. Arab Journal of Urology, 16(1), 113-124. https://doi.org/10.1016/j. aju.2017.11.013

Mendis-Handagama, S., Zirkin, B., Scallen, T., \& Ewing, L. (1990). Studies on peroxisomes of the adult rat leydig cell. Journal of Andrology, 11(3), 270-278.

Miller, N. J., Rice-Evans, C., Davies, M. J., Gopinathan, V., \& Milner, A. (1993). A novel method for measuring antioxidant capacity and its application to monitoring the antioxidant status in premature neonates. Clinical Science, 84(4), 407-412. https://doi.org/10.1042/ cs0840407

Mruk, D., Cheng, C.-H., Cheng, Y.-H., Mo, M., Grima, J., Silvestrini, B., ... Cheng, C. Y. (1998). Rat testicular extracellular superoxide dismutase: Its purification, cellular distribution, and regulation. Biology of Reproduction, 59(2), 298-308.

Opuwari, C., \& Henkel, R. (2016). An update on oxidative damage to spermatozoa and oocytes. BioMed Research International, 2016, 1-11. https://doi.org/10.1155/2016/9540142

Ozturk, U., Ozdemir, E., Buyukkagnici, U., Dede, O., Sucak, A., Celen, S., \& Imamoglu, M. A. (2012). Effect of spermatic vein ligation on seminal total antioxidant capacity in terms of varicocele grading. Andrologia, 44(SUPPL.1), 199-204. https://doi. $\operatorname{org} / 10.1111 / \mathrm{j} .1439-0272.2011 .01164 . x$

Pahune, P., Choudhari, A., \& Muley, P. (2013). The total antioxidant power of semen and its correlation with the fertility potential of human male subjects. Journal of Clinical and Diagnostic Research, 7(6), 991995. https://doi.org/10.7860/JCDR/2013/4974.3040

Pasqualotto, F., Sharma, R., Kobayashi, H., Nelson, D., Thomas, A., \& Agarwal, A. (2001). Oxidative stress in normospermic men undergoing infertility evaluation. Journal of Andrology, 22(2), 316-322.

Pasqualotto, F., Sharma, R., Pasqualotto, E., \& Agarwal, A. (2008). Poor semen quality and ROS-TAC scores in patients with idiopathic infertility. Urologia Internationalis, 81(3), 263-270. https://doi. org/10.1159/000151401 
Pasqualotto, F., Sharma, R., Potts, J., Nelson, D., Thomas, A., \& Agarwal, A. (2000). Seminal oxidative stress in patients with chronic prostatitis. Urology, 55(6), 881-885. https://doi.org/10.1016/S0090 -4295(99)00613-5

Peltola, V., Huhtaniemi, I., \& Ahotupa, M. (1992). Antioxidant enzyme activity in the maturating rat testis. Journal of Andrology, 13(5), 450-455.

Powell, S. (2000). The antioxidant properties of Zinc. The Journal of Nutrition, 130(5S Suppl), 1447S-1454S. https://doi.org/10.1093/ jn/130.5.1447S

Prior, R., \& Cao, G. (1999). In vivo total antioxidant capacity: Comparison of different analytical methods. Free Radical Biology and Medicine, 27(11-12), 1173-1181.

Raigani, M., Yaghmaei, B., Amirjannti, N., Lakpour, N., Akhondi, M. M., Zeraati, H., ... Sadeghi, M. R. (2014). The micronutrient supplements, zinc sulphate and folic acid, did not ameliorate sperm functional parameters in oligoasthenoteratozoospermic men. Andrologia, 46(9), 956-962. https://doi.org/10.1111/and.12180

Rao, A., Ray, M., \& Rao, L. (2006). Lycopene. Advances in Food and Nutrition Research, 51, 99-164.

Rhemrev, J., Van Overveld, F., Haenen, G., Teerlink, T., Bast, A., \& Vermeiden, J. (2000). Quantification of the nonenzymatic fast and slow TRAP in a postaddition assay in human seminal plasma and the antioxidant contributions of various seminal compounds. Journal of Andrology, 21(6), 913-920.

Roychoudhury, S., Sharma, R., Sikka, S., \& Agarwal, A. (2016). Diagnostic application of total antioxidant capacity in seminal plasma to assess oxidative stress in male factor infertility. Journal of Assisted Reproduction and Genetics, 33(5), 627-635. https://doi.org/10.1007/ s10815-016-0677-5

Said, T. M., Kattal, N., Sharma, R. K., Sikka, S. C., Thomas, A. J., Mascha, E., \& Agarwal, A. (2003). Enhanced chemiluminescence assay vs colorimetric assay for measurement of the total antioxidant capacity of human seminal plasma. Journal of Andrology, 24(5), 676-680. https:// doi.org/10.1002/j.1939-4640.2003.tb02726.x

Saleh, R. A., Agarwal, A., Sharma, R. K., Nelson, D. R., \& Thomas, A. J. (2002). Effect of cigarette smoking on levels of seminal oxidative stress in infertile men: A prospective study. Fertility and Sterility, 78(3), 491-499. https://doi.org/10.1016/S0015-0282(02)03294-6

Saleh, R. A., Agarwal, A., Sharma, R. K., Said, T. M., Sikka, S. C., \& Thomas, A. J. (2003). Evaluation of nuclear DNA damage in spermatozoa from infertile men with varicocele. Fertility and Sterility, 80(6), 1431-1436. https://doi.org/10.1016/S0015-0282(03)02211-8

Schwaab, V., Baud, E., Ghyselinck, N., Mattei, M. G., Dufaure, J. P., \& Drevet, J. R. (1995). Cloning of the mouse gene encoding plasma glutathione peroxidase: Organization, sequence and chromosomal localization. Gene, 167(1-2), 25-31. https://doi.org/10.1016/03781119(95)00551-X

Sharma, R. K., Pasqualotto, F. F., Nelson, D. R., Thomas, A. J., \& Agarwal, A. (1999). The reactive oxygen species - Total antioxidant capacity score is a new measure of oxidative stress to predict male infertility. Human Reproduction, 14(11), 2801-2807. https://doi.org/10.1093/ humrep/14.11.2801

Sies, H. (1993). Strategies of antioxidant defense. European Journal of Biochemistry, 215(2), 213-219. https://doi. org/10.1111/j.1432-1033.1993.tb18025.x

Subramanian, V., Ravichandran, A., Thiagarajan, N., Govindarajan, M., Dhandayuthapani, S., \& Suresh, S. (2018). Seminal reactive oxygen species and total antioxidant capacity: Correlations with sperm parameters and impact on male infertility. Clinical and Experimental Reproductive Medicine, 45(2), 88-93. https://doi.org/10.5653/ cerm.2018.45.2.88

Suleiman, S. A., Elamin Ali, M., Zaki, Z. M. S., El-Malik, E. M. A., \& Nasr, M. A. (1996). Lipid peroxidation and human sperm motility: Protective role of vitamin E. Journal of Andrology, 17(5), 530-537.

Valko, M., Izakovic, M., Mazur, M., Rhodes, C. J., \& Telser, J. (2004). Role of oxygen radicals in DNA damage and cancer incidence. Molecular and Cellular Biochemistry, 266(1-2), 37-56.

Vassalle, C., Masini, S., Carpeggiani, C., L'Abbate, A., Boni, C., \& Zucchelli, G. C. (2004). In vivo total antioxidant capacity: Comparison of two different analytical methods. Clinical Chemistry and Laboratory Medicine, 42(1), 84-89.

Vatannejad, A., Tavilani, H., Sadeghi, M. R., Amanpour, S., Shapourizadeh, S., \& Doosti, M. (2017). Evaluation of ROS-TAC score and DNA damage in fertile normozoospermic and infertile asthenozoospermic males. Urology Journal, 14(1), 2973-2978.

WHO (2010). WHO laboratory manual for the Examination and processing of human semen (5th ed.). Geneva, Switzerland: WHO.

Zini, A., \& Schlegel, P. (1997). Identification and characterization of antioxidant enzyme mRNAs in the rat epididymis. International Journal of Andrology, 20(2), 86-91.

How to cite this article: Gupta S, Finelli R, Agarwal A, Henkel

R. Total antioxidant capacity-Relevance, methods and clinical implications. Andrologia. 2021;53:e13624. https://doi. org/10.1111/and.13624 